\title{
Selected Topics on Enterprise and Automated Systems: Editorial Introduction to Issue 4 of CSIMQ
}

\author{
Raimundas Matulevičius ${ }^{1}$, Thorsten Weyer $^{2}$ \\ ${ }^{1}$ Institute of Computer Science, University of Tartu, Tartu, Estonia \\ ${ }^{2}$ paluno - The Ruhr Institute for Software Technology, University of Duisburg-Essen, Germany \\ Raimundas.Matulevicius@ut.ee, Thorsten.Weyer@paluno.uni-due.de
}

Nowadays enterprise and automated systems play an important role in different business, physical, and social contexts. The fourth issue of the journal Complex Systems Informatics and Modeling Quarterly suggests five challenging studies in this area addressing stakeholder personalities, enterprise actions, business process OR-joint semantics, validation of automated plant control, and safety analysis in complex embedded systems.

Development of any type of software systems involves a number of various stakeholders having different goals, interests, and personal profiles. In their paper on "Relationship between Personality Types Conceptualized by C. G. Jung and Latvian IT Specialist Preferences", M. Dargis et al. apply the Myers-Briggs Type Indicator (MBTI), a personality profiling tool, to conduct a survey on the performance of IT stakeholders. The survey results show Latvian IT developer personality traits, which let to assume that targeted project management skills, motivational models, and technical skills are used and have to be provided to ensure successful teamwork in IT projects performed in enterprises of Latvia and other countries.

Enterprise modeling consists of different types of actions, which all together contribute to the enterprise model and characterize enterprise's goals, needs, and strategies. In the paper "The Diversity of Enterprise Modeling - a Taxonomy for Enterprise Modeling Actions", U. Seigerroth presents taxonomy for enterprise modeling actions. The taxonomy classifies the actions along the hierarchy and process dimensions. The taxonomy helps to increase stakeholder understanding and awareness about the enterprise modeling actions and contributes to the high degree of business and IT alignment.

Contribution by T. Prinz and W. Amme continues the topic on enterprise modeling. More specifically, the authors focus on the semantics of the business process modeling. The paper on "A Complete and the Most Liberal Semantics for Converging OR Gateways in Sound Processes" considers the semantics of the OR gateways (OR-joint). The correct expression of these gateways still remains a challenging task in defining an automated and correct execution of processes. The study illustrates that OR-joints could result in a deadlock in unsound workflow graphs. To solve this problem, authors introduce a new definition of soundness of workflow graphs with arbitrary OR-joint semantics through presentation of completeness criterion. Thus, the study suggests the way to detect such failure situations using static analysis methods.

The topic of process automation is also considered in paper on "Early Validation of Automation Plant Control Software using Simulation Based on Assumption Modeling and Validation Use Cases" by V. Brandstetter at al. Automation control systems are software systems that calculate functions, solve equations, and control devices. The authors. present an approach to validate automation control systems using technical plant process and design at early stages of development. The validation is performed through simulation of modeled assumptions 
and validation use cases. The authors also discuss that their proposed approach could be used to validate software systems with complex physical and technical constraints such as embedded systems, which increasingly are growing in their complexity.

In the paper on "Contract-based Design of Embedded Systems Integrating Nominal Behavior and Safety", B. Kaiser et al. propose a component-based approach to bridge the gab between the nominal embedded system functions and safety development. The paper introduces a design and safety process for complex embedded systems. Authors suggest decomposing system to assess the criticality of component failures. Then, the safety requirements to cope with consequences of these failures are introduced. The particularity of the proposal is that the approach is guided by the contract violations as formal definitions for failure types.

The CSIMQ editorial team is thankful to all authors, who submitted their papers to this issue. We also appreciate very much the excellent contribution of reviewers providing valuable comments and improvement proposals for the submitted papers. 